\title{
Hormonal Dysfunctioning in Thyroidectomy Patients
}

\author{
Muhammad Ameen* \\ Department of Zoology/ Biology, Pakistan \\ *Corresponding author: Muhammad Ameen, Department of Zoology/ Biology, Rawalpindi, Pakistan
}

\begin{tabular}{l} 
ARTICLE INFO \\
Received: 仹 April 11, 2019 \\
Published: 幽 April 29, 2019 \\
\hline
\end{tabular}

Citation: Muhammad Ameen. Hormonal Dysfunctioning in Thyroidectomy Patients. Biomed J Sci \& Tech Res 17(4)2019. BJSTR. MS.ID.003022.

Abbreviations: T3: Triiodothyronine; NORI: Nuclear Medicine Oncology and Radiotherapy Institute; TSH: Thyroid Stimulating Hormone; T4: Etraiodothyronine; ECLIA: Electro Chemiluminescent Immunoassays; TFTs: Thyroid Functions Tests; NG: Nodular Goiter; MNG: Multinodular Goiter

\begin{abstract}
A thyroidectomy is a surgical technique that is used to remove the thyroid gland. It is used to treat hyperthyroidism, noncancerous enlargement of the thyroid (goiter) and cancer. Partial thyroidectomy is done in order to remove part of thyroid gland and thyroid may be able to function normally after surgery. While in case of (total thyroidectomy) entire thyroid is removed and it requires daily treatment with thyroid hormone to replace your thyroid's natural function. Thyroid disorder is physical ailment that arises from its dysfunctioning that produces triiodothyronine (T3) and thyroxin (T4). Both hypothyroid and hyperthyroid patients have higher and lower concentration of TSH respectively. The appropriate study has incorporated 26 patients of thyroidectomy as experimental group and 50 control individuals. Thyroid scan, physical examination and ultrasound examination are the techniques that are used to diagnose patients. These have evaluated through various statistical techniques in order to investigate thyroid dysfunctioning. Hypothyroidism and hyperthyroidism can be accurately diagnosed with laboratory tests performed at Nuclear Medicine, Oncology and Radiotherapy Institute (NORI). The data along with detailed history were collected with the help of clinicians in Nuclear Medicine, Oncology and Radiotherapy Institute (NORI) and entered in the Performa. The diagnostic evaluation relating to functional and morphological characterization has examined through serum TSH concentration and imaging. The results have determined non-significant difference between hypothyroid and hyperthyroid patients of thyroidectomy patients and control group individuals. The study has concluded that the prevalence of goiter may be dependent on the higher and lower concentration of thyroid stimulating hormone (TSH).
\end{abstract}

\section{Introduction}

The highly vascular endocrine gland the thyroid gland lying in the neck in front of trachea extending from the fifth cervical to the first thoracic vertebrae [1]. Lang and Lo CY [2] have reported that total thyroidectomy is done when disease has invaded in both thyroid lobes (i.e. bilateral multinodular goiter). Hauch et al. [3] have determined that there was a significantly increased risk of complications after total thyroidectomy compared to lobectomy (20.4 vs. $10.8 \%$ : p<0.0001) in the adult patients. Evered et al. [4] have investigated the normal values of T3 and T4 and high range of TSH at the state of mild hypothyroidism. Vander Pump [5] has reported that the enlargement of whole thyroid gland in case of diffuse goiter at the state of both hypothyroidism and hyperthyroidism is observed at the age of puberty and toxic diffuse goiter is also known as grave's disease is more prevalent in hyperthyroid patients. Kumar et al. [6] have reported that diffuse goiter (Simple) or physiological goiter is the most common thyroid disorder among different thyroid diseases.

They have further investigated that diffuse goiter has been exhibiting equivalence with diffuse radioactive iodine uptake, while multinodular goiter has not been exhibiting equivalence with irregularity in iodine uptake and activity throughout the thyroid gland. Schwartz et al. [7] have observed the follicular cells of the thyroid gland have produced and have secreted the two basic forms of thyroid hormone that are T4 (3,5,3',5'-tetraiodothyronine) and T3 (3, 3',5-triiodothyronine). They have further revealed that these hormones have played extremely important role in the normal development of cellular metabolism, cell proliferation and cell differentiation. Surks et al. [8] have described that a thyroid disorder is physical ailment that arises from its dysfunctioning that produces triiodothyronine (T3) and thyroxin (T4). They have 
further observed that this dysfunctioning have influenced functions of the pituitary gland and hypothalamus and consequently their secretions. Oppenheimer et al. [9] have revealed that tetraiodothyronine (T4) is generated by the thyroid gland as an inactive compound.

They have further described that this is transformed into the tissues to triiodothyronine (T3) that combines to the nuclear receptors in order to initiate thyroid hormone (TH) action. Guyton and Hall. (1996) have analyzed that thyroid gland have produced about 7\% triiodothyronine (T3) and about 93\% thyroxin (T4). Triiodothyronine (fT3) has 3-5 folds greater biological potency than thyroxin (fT4). Morreale et al. [10] have reported that thyroid hormones as being critical regulatory molecules have regulated normal brain functioning during development of vertebrate brain. They have further examined that these are Tyrosine based hormones that are partially composed of iodine and their deficiency enlarges the thyroid tissue which is known as simple goiter Suzuki et al. $[11,12]$ have examined that alteration in TSH and free thyroid hormones have been observed in gender and during aging. They have further investigated that in males the process of aging have restrained the free thyroid hormones concentration but have not influenced on the concentration of TSH. While, in females the free thyroid hormones levels have not been changed with aging but TSH level have been increased in age-dependent areas of severe iodine deficiency can be as high as $80 \%$.

Khan et al. [13] have observed that two major ailments of thyroid gland, one is higher generation of thyroid hormones that is called hyperthyroidism and other is lower generation of thyroid hormones that is called hypothyroidism. Khan et al. [13] also have examined that serum TSH level has been elevated in case of primary hypothyroidism but values of $\mathrm{T} 3$ and $\mathrm{T} 4$ have remained below the normal range. Evered et al. [14] have been investigated that in case of mild hypothyroidism serum TSH also have been observed high and the values of T3 and T4 have remained in normal range. Caldwell et al. 1985 have been reported that in case of hyperthyroidism the values of triiodothyronine and Thyroxin have been observed above high level and TSH has subdued due to negative feedback mechanism. The objective of study is to compare the hormonal profile of subclinical and clinical hypothyroid and hyperthyroid patients with normal healthy individuals and will be evaluated statistically. Various medical disorders and abnormalities in thyroid hormones will be explored in Pakistani population. Further, profile of thyroid hormones will be analyzed via level of different hormones and antibodies like TSH, FT4, T3 and ant thyroglobulin respectively will be analyzed from serum samples of patients. Analytical evaluation of the hormonal profile of patient will be carried out via electro chemiluminescent immunoassays (ECLIA).

\section{Materials and Methods}

Thyrodectomy patients of goiter versus normal individuals were statistically evaluated at Nuclear Medicine, Oncology and
Radiotherapy Institute (NORI), Islamabad. Approximately 26 patients of thyrodectomy and 50 normal individuals were included in this appropriate study. The whole population was categorized into two major groups, one is experimental group and other is control group. The blood tests reports showing normal values of thyroid functions tests (TFTs) were designated as normal while patients having not normal values of thyroid functions tests (TFTs) were designated as abnormal. The experimental group constituted 26 patients of goiter and 50 individuals as a control group. In a whole sample of population 30 were observed as normal and 46 as abnormal patients. The abnormal patients were further categorized into hyperthyroid and hypothyroid according to the values of TSH levels. Hypothyroidism and hyperthyroidism can be accurately diagnosed with laboratory tests performed at NORI. The patients having higher concentration of TSH were designated as hypothyroid and patients having low concentration of TSH were designated as hyperthyroid.

Individuals having no goiter were taken as control group, but they can have hypothyroidism or hyperthyroidism. The patients having diffuse goiter were diagnosed through physical examination, clinical examination, Thyroid Scan, and Ultrasound reports. The blood reports of thyroid function tests were collected from various laboratories of Pakistan and normal TSH concentration $(0.27$ to $4.2 \mu \mathrm{IU} / \mathrm{ml}$ ) was kept as criteria for the diagnosis of thyroid dysfunction.

Setting and Study Area: The present study was carried out in Nuclear Medicine, Oncology and Radiotherapy Institute (NORI).

Study Population: Total 76 patients that were referred for thyroid scan were included in the study.

Inclusion Criteria: All patients having goiter in any age group will be included in the study.

\section{Exclusion criteria:}

a) Pregnancy

b) Liver disease as evidenced by raised ALT level

c) Drugs: Amiodarone, lithium, anti-thyroid medications (neomercazole/procarbizole), thyroxin

Study Group: Thyroidectomy patients of goiter were based on the incidence of thyroid functions tests (TFTs) constituting the whole population sample.

Data Collection: After having informed consent (Annexure-I) from patients, the data along with detailed history was collected with the help of clinicians in NORI and entered in the Performa (Annexure-II).

Data Analysis: The data were analyzed using SPSS version 17. The average values for age, history of goiter (duration) etc. were calculated. The percentage of different thyroid dysfunctions 
(hypothyroid, hyperthyroid) in patients of diffuse goiter was analyzed through confidence interval (0.05). Statistically defined P-value of the study is $(0.000)$. The study has considered thyroid stimulating hormone (TSH) as a criterion for the diagnosis of thyroid dysfunction. The normal range of thyroid function (TFTs) regarding TSH diagnosed at NORI hospital is (0.27-4.2 $\mu \mathrm{IU} / \mathrm{ml})$ and other clinical laboratories of Pakistan. The patients having high TSH concentration have been designated as Hypothyroid and the patients having low serum TSH concentration have been designated as hyperthyroid while the patients having normal TSH values are considered as euthyroid.

\section{Results}

The current paper has investigated that $60 \%$ of goiter patients have abnormal TSH concentration, while $40 \%$ have normal TSH concentration in a whole sample of population. Hyperthyroid patients with low TSH levels have constituted about 26\% while hypothyroid with high TSH levels have constituted $74 \%$ proportion of whole population sample. TSH levels ranging from $0.27-4.2 \mu \mathrm{IU} /$ $\mathrm{ml}$ was considered as normal for the diagnosis in thyroid function tests (TFTs). When the abnormal patients of thyroidectomy patients having the clinical symptoms of hypothyroid or hyperthyroid were compared with control group individuals, statistically nonsignificant difference was observed in this case. Comparatively, similar results were obtained in the studies of nodular goiter and multinodular goiter. The study has worked out to determine the comparative evaluation of thyroid function tests between normal and abnormal, hyperthyroid and hypothyroid of diffuse goiter, nodular goiter (NG), multinodular goiter (MNG) and control group. The statistical evaluation of thyroid function tests in the group having diffused goiter has done in the statistical evaluation tables.

\section{Discussion}

The statistical difference between hypothyroid and hyperthyroid of thyrodectomy patients versus control individuals have demonstrated in Table 1. Both hyperthyroid and hypothyroid patients have proportion of (60\%) and euthyroid have constituted (40\%) of proportion in whole sample of population. Moreover, among abnormal patients $71 \%$ having low TSH levels and $29 \%$ having high TSH levels. They have revealed the fact that goiter is dependent on the concentration of TSH. The patients with low TSH levels have greater chance of occurrence of disease as compared to patients with high TSH levels. When the lipid profile of euthyroids clinical and subclinical hypothyroid and hyperthyroid versus control group individuals were compared, statistically nonsignificant difference was observed by non-parametric tests ( $p$ $>0.05$ ). Studies pertaining to nodular goiter and multinodular goiter have shown similar results. Hypothyroid, euthyroids, Clinical and subclinical hypothyroid and hyperthyroid patients of diffuse goiter and multinodular goiter (MNG) have not altered lipid profile of control individuals contrary to euthyroids of nodular goiter. However, thyroid dysfunctioning was not prominent in patients experiencing diffuse goiter and multinodular goiter (MNG).

Table 1: X2 Calculations (Two-way Contingency) showing statistical difference between hypothyroid, hyperthyroid and normal TSH Patients of thyroidectomy patients versus control individuals.

\begin{tabular}{|c|c|c|c|c|}
\hline \multirow{2}{*}{ Number of patients with TSH concentration } & & & & \multirow{2}{*}{ Total } \\
\hline & Male & Female & Control & \\
\hline \multirow{3}{*}{ Number of patients with normal TSH concentration } & 3 & 3 & 3 & \multirow{3}{*}{30} \\
\hline & 4.34 & 4.34 & 4.34 & \\
\hline & $(0.41)$ & $(0.41)$ & $(0.41)$ & \\
\hline \multirow{3}{*}{ Number of patients with high TSH concentration } & 4 & 8 & 22 & \multirow{3}{*}{34} \\
\hline & 4.92 & 6.71 & 22.37 & \\
\hline & $(0.17)$ & $(0.25)$ & $(0.01)$ & \\
\hline \multirow{4}{*}{ Number of patients with low TSH concentration } & 4 & 3 & 5 & \multirow{3}{*}{12} \\
\hline & 1.74 & 2.37 & 7.89 & \\
\hline & $(2.95)$ & $(0.17)$ & $(1.06)$ & \\
\hline & 11 & 15 & 50 & 76 \\
\hline
\end{tabular}

The result revealed that TSH, FT3 and FT4 levels did not affect the onset goiter in both groups. Similarly, the study is correlated with the findings of Kumar et al. [15] investigated that diffuse goiter has been exhibiting equivalence with diffuse radioactive iodine uptake. The prevalence of disease is not dependent upon the concentration of TSH. Therefore, the research work has inconsistency with the findings of Dr. Firdushi Begum [16] that estimation of serum thyroid hormones and TSH concentrations has great significance for the diagnosis of thyroid problem. The current study has rejected the serum TSH concentration usefulness in determining thyroid problem, on this ground it is not related to the findings of Dr. Firdushi Begum [18] The recent study is also not according to the findings of Ladenson et al. [17] have also considered that the TSH test as a criterion for the diagnosis of thyroid dysfunction, especially in cases of minimal thyroid failure (subclinical hypothyroidism), while such results are rejected in this new findings. The study has incon- 
sistency with the findings of Evered et al. [19] have been investigated that in case of mild hypothyroidism serum TSH also have been observed high and the values of T3 and T4 have remained in normal range, while in case of present study serum TSH levels fluctuates. Moreover, current new findings have already challenged the published results. Such studies would be helpful to understand on the prevalence of various types of goiter in different subjects and will also suggest the measures to minimize the goiter associated with its onset. It is further suggested that that the role of hormonal interactions in these patients may also be investigated with reference to their different metabolic disorders.

\section{Conclusion}

The present study concluded that prevalence of goiter may be dependent on the extent of hormonal dysfunctioning specifically normal and abnormal TSH levels. In future comprehensive investigation is warrant covering the epidemiological profile of the patients having goiter.

\section{References}

1. Dixit D, Shilpa MB, Harsh MP, Ravishankar MV (2009) Agenesis of isthmus of thyroid gland in adult human cadavers: a case series. Cases J 2: 6640 .

2. Lang BH, Lo CY (2005) Total thyroidectomy for multinodular goiter in the elderly. Am J Surg 190: 418-423.

3. Hauch A, Al-Qurayshi Z, Randolph G, Kandil E (2014) Total thyroidectomy is associated with increased risk of complications for low- and highvolume surgeons. Ann Surg Oncol 21: 3844-3852.

4. Evered DC, PA Vice, E Green, D Appleton (1976) Assessment of thyroid hormone assays. From the Endocrine Unit, The Royal Victoria Infirmary, Newcastle upon Tyne. J clin Path (29): 1054-1059.

5. Vander Pump MPJ (2005) The epidemiology of thyroid diseases. In: LE B and Utiger RD (ed.) Werner and Ingbar's the thyroid: a fundamental and clinical text ( $9^{\text {th }}$ edn.). Philadelphia: JB Lippincott Raven pp: 398-496.

6. Schwartz CE, Stevenson RE (2007) The MCT8 thyroid hormone transporter and Allan-Herndon-Dudley syndrome. Best Pract Res Clin Endocrinol Metab 21: 307-321.

\section{ISSN: 2574-1241}

DOI: 10.26717/BJSTR.2019.17.003022

Muhammad Ameen. Biomed J Sci \& Tech Res

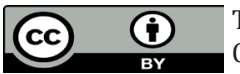

This work is licensed under Creative Commons Attribution 4.0 License

Submission Link: https://biomedres.us/submit-manuscript.php
7. Surks MI, Hollowell JG (2007) Age-specific distribution of serum thyrotropin and antithyroid antibodies in the US population: implications for the prevalence of subclinical hypothyroidism. J Clin Endocrinol Metab 92: 4575-4582.

8. Oppenheimer JH, Koerner D, Schwartz HL, Surks MI (1972) Specific nuclear triiodothyronine binding sites in rat liver and kidney. J Clin Endocrinol Metab 35: 330-333.

9. Morreale de Escobar G, Obregon MJ, Escobar Del Rey F (2004) Role of thyroid hormone during early brain development. Eur J Endocrinol 151: 45-78.

10. Suzuki S, Nisiho S, Takeda T, Komatsu M (2012) Gender-specific regulation of response to thyroid hormone in aging. Thyroid Res 5: 34 45.

11. Khan A, Khan MAM, Akhtar S (2002) Thyroid disorders, etiology and prevalence. Med Sci 2: 89-94.

12. Kumar V, Cotran RS, Robbins SL (1997) Basic pathdogy ( $6^{\text {th }}$ edn.). WB Saunders and co. pp. 643-652.

13. Firdushi Begum (2015) Scholars Journal of Applied Medical Sciences. Estimation of TSH \& Thyroid Hormones. Sch J App Med Sci 3(8E): 30963102.

14. Ladenson PW, PA Singer, KB Ain, N Bagchi, ST Bigos, et al. (2000) American Thyroid Association guidelines for detection of thyroid dysfunction. Arch Intern Med (160): 1573-1575.

15. Caldwell G, Gow SM, Sweating VM, Kellett HA, Beckett HJ (1985) A new strategy for thyroid function testing. Lancet 1: 1117-1119.

16. Guyton AC, TE Hall (1996) Text Book of Medical Physiology. Pp. 945-946.

17. Table (1) $\mathrm{X}^{2}$ Calculations (Two-way Contingency) showing statistical difference between hypothyroid, hyperthyroid and normal TSH Patients of diffuse goiter versus control individuals.

18. Table (2) $\mathrm{X}^{2}$ Calculations (Two-way Contingency) showing statistical difference between hypothyroid, hyperthyroid and normal TSH Patients of nodular goiter (NG) versus control individuals.

19. Table (3) $X^{2}$ Calculations (Two-way Contingency) showing statistical difference between hypothyroid, hyperthyroid and normal TSH Patients of multinodular goiter (MNG) versus control individuals.

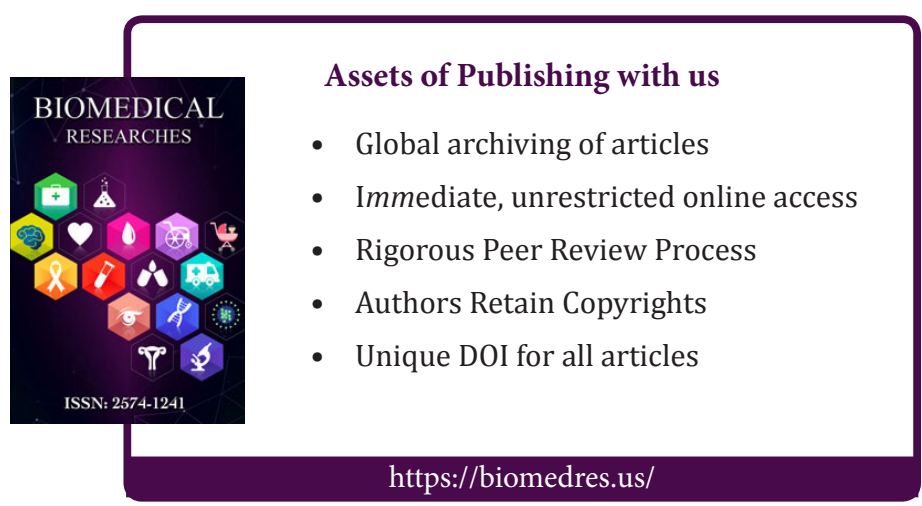

